\title{
The Effect of Aromatherapy with Lavender Oil on the Health-Related Quality of Life in Patients with Fibromyalgia
}

\author{
Gulsah Yasa Ozturk ${ }^{1}{ }^{1}$ and Ibrahim Bashan $\mathbb{D}^{2}$ \\ ${ }^{1}$ Physical Medicine and Rehabilitation Department, Adana City Hospital, Adana 01230, Turkey \\ ${ }^{2}$ Department of Medical Education, Medical Faculty of Mersin University, Mersin 33343, Turkey \\ Correspondence should be addressed to Ibrahim Bashan; ibashan@yahoo.com
}

Received 10 March 2021; Revised 3 April 2021; Accepted 21 April 2021; Published 28 April 2021

Academic Editor: Nesibe E. Kafkas

Copyright (c) 2021 Gulsah Yasa Ozturk and Ibrahim Bashan. This is an open access article distributed under the Creative Commons Attribution License, which permits unrestricted use, distribution, and reproduction in any medium, provided the original work is properly cited.

\begin{abstract}
The aim of this study was to evaluate the effect of lavender oil, which has been used in complementary medicine as an aromatherapeutic, on the health-related quality of life in patients diagnosed with fibromyalgia and to determine the main volatile compounds of lavender oil with healing effects. Forty-two patients aged 18 years and over who were diagnosed with fibromyalgia according to the American College of Rheumatology 2010 diagnostic criteria and used lavender oil as an aromatherapeutic by applying it on the wrist or via inhalation for four weeks were included in this study. The Short-Form (SF)-36 Quality of Life Scale was administered to the patients at baseline and after four weeks of lavender oil treatment to determine whether there was any change in their quality of life. The averages and correlation levels between the scores were calculated with the paired-sample $t$-test taking $p<0.05$ as the statistical significance limit. In addition, the chromatographic analysis of lavender oil was performed with the combined headspace solid-phase microextraction and gas chromatography-mass spectrometry methods. When the quality of life scores were evaluated at baseline and after four weeks of lavender oil treatment, improvements were detected in all the subscales of SF-36 at the end of the study. It was found that all the subscale scores, except for general health, were statistically significantly higher compared to the baseline scores $(p<0.05)$. The major volatile compounds detected in lavender oil were linalool $(29.33 \%)$ and linalyl acetate (27.69\%). The remaining main compounds were terpinolene, cis-ocimene, eucalyptol, and lavandulol in the order of frequency, which is consistent with the literature. To our knowledge, this is the first study investigating the effect of lavender oil aromatherapy on patients diagnosed with fibromyalgia, and our results support that lavender oil aromatherapy significantly improves the quality of life in these patients. However, further comprehensive studies are needed to confirm our findings.
\end{abstract}

\section{Introduction}

Fibromyalgia is a chronic pain syndrome that is more common in adults between the ages of 20 and 55 years and especially in women, with the prevalence ranging from 2 to $8 \%$ in the general population. Common musculoskeletal pain, sleep disorders, and fatigue are often found together [1]. Despite various complaints of patients, no significant impairment is found in physical examination and laboratory tests, suggesting that these symptoms may be psychological [2]. Psychological factors are an important component of all types of pain, especially chronic pain, and they create an interaction in a vicious circle [3].
It is known that the quality of life of individuals with fibromyalgia syndrome is affected negatively [4]. Widespread pain, fatigue, sleep disorders, and psychiatric symptoms are effective in deteriorating the quality of life, cause loss of labor force, and result in high health-related expenses. The main goal in the treatment of the disease is to increase the quality of life by reducing pain [5]. Quality of life is a general parameter that shows the comfort levels of individuals, and health-related quality of life scales are widely used in evaluating the effects of many chronic diseases, including fibromyalgia, on individuals [6-8]. The Short-Form Quality of Life Scale (SF-36), adapted into Turkish by Koçyiğit et al., has been proven to be reliable and 
valid and is one of the frequently used quality of life scales in the assessment of health-related quality of life. It consists of 36 items structured under eight subscales. The distribution of the items according to the subscales is as follows: physical functionality (10 items), social functionality (two items), role limitations due to physical functionality (four items), mental role (emotional problems) (three items), mental health (five items), vitality (four items), body pain (two items), and general health (six items). Separate scores are obtained from each subscale, with a high score indicating good health in the corresponding subscale. Each subscale is evaluated separately, and it is not possible to calculate the total score for the whole scale $[9,10]$.

Analgesics, muscle relaxants, and anxiolytics are generally used to reduce the complaints caused by fibromyalgia [11]. However, studies have also reported that these drugs may cause side effects in some individuals [12]. Due to these side effects, patients sometimes refer to complementary medicine approaches, such as nonpharmacological exercise, mind and body therapy, acupuncture, massage therapy, and aromatherapy [13]. Lavender oil, an aromatherapeutic agent, is also increasingly used for this purpose due to its analgesic, anti-inflammatory, and anxiolytic effects [14]. Lavender oil is considered to physically and psychologically calm the individual and relieve anger and fatigue [15]. The flowers' leaves and oil of lavender (Lavandula angustifolia) contain linalyl acetate, linalool, perillyl alcohol, 1,8-cineole (eucalyptol), and at least 100 other known compounds. It is recognized as "generally safe" to be consumed as food by the US Food and Drug Administration [16].

This study aimed to evaluate the effect of lavender oil, which has been used in complementary medicine (aromatherapy) for many years and recently become more popular, on the health-related quality of life in patients diagnosed with fibromyalgia and to determine the volatile compounds of lavender oil with healing effects.

\section{Materials and Methods}

Male and female patients aged 18 years and over who presented to the physical therapy and rehabilitation outpatient clinic of Adana City Hospital in the second half of 2019 due to widespread pain and were diagnosed with fibromyalgia based on the American College of Rheumatology 2010 fibromyalgia diagnostic criteria (FMS ACR) [17] were included in this study. Patients with any chronic disease, pregnant and breastfeeding women, patients with a history of medical treatment for fibromyalgia, those that used medical treatment together with aromatherapy, and those that used drugs for sleeping disorders during and/or before applying lavender oil were excluded from the study. Forty-two patients who used aromatherapeutic lavender oil (product license/permit approval code: E-97834074-3992344860-Istanbul Provincial Directorate of Agriculture) by applying it on the wrist and via inhalation for four weeks were evaluated. The mixture, prepared by dropping $1 \mathrm{ml}$ of essential lavender oil in $10 \mathrm{ml}$ of fixed oil, was applied (three drops) on the wrist and inhaled for 10 minutes, keeping it about $10 \mathrm{~cm}$ away from the nose. This intervention was recommended for each patient once a day before going to bed in the evening, and all subjects noted that they received the intervention as recommended. The SF-36 Quality of Life Scale was administered to the patients to determine their experiences before and after four weeks of using lavender oil via face-to-face (during follow-up visits) or phone interviews. The SF-36 scores were calculated by scoring the items in eight subscales from 0 to 100 , and the obtained data were statistically analyzed using SPSS (version 21). The averages and correlation levels between the scores were calculated with the paired-sample $t$-test taking $p<0.05$ as the statistically significant limit. This study was approved by the Adana City Hospital Clinical Ethic Board.

In Cukurova University, Department of Horticulture, the chromatographic analysis of lavender oil was performed with the combined headspace solid-phase microextraction (HS/SPME) and gas chromatography-mass spectrometry (GC/MS) methods for the determination of the volatile compounds of lavender oil. The extraction of essential oils from lavender oil was performed by treating the oil with $5 \mathrm{M}$ $\mathrm{CaCl}_{2}$ on a magnetic stirrer at $3^{\circ} \mathrm{C}$ for 20 minutes in a standard headspace glass bottle (Supelco, $75 \mathrm{~mm} \times 23 \mathrm{~mm}$ ). Analyses were undertaken with three replications. The volatiles were absorbed by polydimethylsiloxane (PDMS) using an SPME needle (Supelco, Bellefonte, PA). PerkinElmer GC (Clarus 600) equipped with HP-5 MS $(30 \mathrm{~m} \times 0.25 \mathrm{~mm} \times 0.25 \mu \mathrm{m})$ and a fused-silica capillary column were used for the separation of volatiles. The carrier gas was helium $(0.6 \mathrm{ml} / \mathrm{min})$. The injection temperature was set as $280^{\circ} \mathrm{C}$. The initial column heating was $40^{\circ} \mathrm{C}$ for two minutes, followed by an increase of temperature to $250^{\circ} \mathrm{C}$ at a rate of $5^{\circ} \mathrm{C} /$ minute, at which the sample was kept for 20 minutes. Compounds were determined by obtaining their mass spectra and using the NIST, Wiley, and flavor libraries according to their retention time.

\section{Results and Discussion}

3.1. Results. Thirty-six of the individuals participating in the study were female $(85.7 \%)$, and six were male $(14.3 \%)$. The mean age of women was 44.6, and that of men was 48.3. When the quality of life scores were evaluated at baseline and after four weeks of lavender oil treatment, improvements (higher scores) were detected in all subscales of SF-36. It was found that all the subscale scores, except for general health, were statistically significantly higher compared to the baseline $(p<0.05)$ (Table 1$)$.

As shown in Table 2, a total of 33 volatile compounds were detected, including two ketones, 10 alcohols, nine esters, and 12 terpenes. Alcohols (40.60\%) formed the largest part of volatiles in lavender oil followed by esters (32.29\%), terpenes $(25.53 \%)$, and ketones $(1.58 \%)$. The major volatile compounds detected in lavender oil were linalool (29.33\%) and linalyl acetate $(27.69 \%)$. The remaining main compounds were terpinolene, cis-ocimene, eucalyptol, and lavandulol in the order of frequency. These compounds are consistent with those previously reported in the literature [18]. 
TABLE 1: Comparison of the quality of life scores at baseline and after four weeks of lavender oil treatment.

\begin{tabular}{|c|c|c|c|c|c|}
\hline Subscales & Baseline & Min-max & After four weeks & Min-max & $p$ \\
\hline General health & $36.10 \pm 20.90$ & $0-75$ & $39.27 \pm 14.60$ & $15-95$ & $>0.05$ \\
\hline Physical functioning & $53.41 \pm 27.44$ & $0-100$ & $75.85 \pm 23.1$ & $30-100$ & $<0.001$ \\
\hline Physical role & $26.83 \pm 38.89$ & $0-100$ & $68.29 \pm 35.81$ & $0-100$ & $<0.001$ \\
\hline Mental role & $24.39 \pm 40.16$ & $0-100$ & $76.42 \pm 30.95$ & $0-100$ & $<0.001$ \\
\hline Social functioning & $40.24 \pm 24.13$ & $0-87.5$ & $64.94 \pm 15.62$ & $37.5-100$ & $<0.001$ \\
\hline Body pain & $35.91 \pm 27.03$ & $0-100$ & $66.71 \pm 18.49$ & $35-100$ & $<0.001$ \\
\hline Mental health & $37.56 \pm 22.00$ & $0-80$ & $70.73 \pm 13.87$ & $32-100$ & $<0.001$ \\
\hline Vitality & $32.20 \pm 21.65$ & $0-80$ & $67.48 \pm 15.00$ & $40-100$ & $<0.001$ \\
\hline
\end{tabular}

TABLe 2: Volatile compounds of lavender oil.

\begin{tabular}{|c|c|c|}
\hline RT & Compound name & $\%$ \\
\hline & Ketones & \\
\hline 2.337 & 2-Propanone (CAS) & 0.92 \\
\hline \multirow{2}{*}{18.259} & Camphor (CAS) & 0.66 \\
\hline & Total ketones & 1.58 \\
\hline & Alcohols & \\
\hline 13.789 & 1-Hexanol (CAS) & 0.25 \\
\hline 14.958 & 3-Octanol (CAS) & 0.62 \\
\hline 16.535 & 1-Octen-3-ol & 1.25 \\
\hline 17.052 & cis-Linalool oxide & 0.40 \\
\hline 19.446 & Linalool & 29.33 \\
\hline 22.453 & Lavandulol & 2.91 \\
\hline 22.93 & Alpha-Terpineol & 1.92 \\
\hline 25.301 & Nerol (CAS) & 0.23 \\
\hline 26.357 & trans-Geraniol & 0.47 \\
\hline \multirow[t]{2}{*}{9.731} & Eucalyptol & 3.22 \\
\hline & Total alcohols & 40.60 \\
\hline & Esters & \\
\hline 6.221 & Acetic acid, butyl ester (CAS) & 0.19 \\
\hline 10.146 & Butanoic acid, butyl ester (CAS) & 0.66 \\
\hline 11.761 & Acetic acid, hexyl ester (CAS) & 0.53 \\
\hline 13.473 & 3-Octanyl acetate & 0.68 \\
\hline 13.62 & Propanoic acid, 2-methyl-, hexyl ester (CAS) & 0.29 \\
\hline 14.74 & Oct-1-en-3-yl acetate & 1.00 \\
\hline 15.756 & Butanoic acid, hexyl ester (CAS) & 0.94 \\
\hline 19.968 & Linalyl acetate & 27.69 \\
\hline \multirow{2}{*}{24.401} & Geranyl acetate & 0.31 \\
\hline & Total esters & 32.29 \\
\hline & Terpenes & \\
\hline 5.002 & Alpha-Pinene & 0.93 \\
\hline 5.13 & 1-Phellandrene & 0.56 \\
\hline 5.879 & Camphene (CAS) & 0.61 \\
\hline 8.114 & Delta-3-carene & 0.41 \\
\hline 8.679 & Beta-Myrcene & 0.58 \\
\hline 9.575 & Bicyclo[2.2.1]hept-2-ene, 1,7,7-trimethyl & 2.50 \\
\hline 10.82 & cis-Ocimene & 3.86 \\
\hline 10.993 & Gamma-Terpinene & 0.45 \\
\hline 11.991 & Alpha-Terpinolene & 0.45 \\
\hline 14.533 & 2,6-Dimethyl-2,4,6-octatriene & 0.38 \\
\hline 20.918 & Terpinolene & 13.59 \\
\hline \multirow[t]{2}{*}{20.234} & Santalene & 1.21 \\
\hline & Total terpenes & 25.53 \\
\hline
\end{tabular}

$\mathrm{RT}$ : retention time.

3.2. Discussion. Fibromyalgia is a common chronic pain syndrome characterized by sleep disturbance, fatigue, and anxiety, and although its cause is not exactly known, it has been suggested in a study that it is caused by a disorder in pain physiology. Fibromyalgia symptoms significantly affect the overall quality of life. Although a complete cure cannot be achieved, the treatment aims to reduce the symptoms and increase the quality of life of patients [19]. Studies have also shown that the aromatherapeutic application of lavender oil is used as a complementary medicine approach to relieve chronic pain, anxiety, and sleep disorders, which are the basic symptoms of fibromyalgia [15, 20-22]. While there is insufficient evidence for the curative effects of lavender oil aromatherapy on acute pain [23-25], there are studies showing that it has curative effects, especially in the case of chronic pain $[26,27]$.

Although the symptoms, causes, and mechanisms of pain differ, they can be evaluated under three main headings: acute pain, inflammatory pain, and neuropathic pain. Neuropathic pain and inflammatory pain can cause chronic pain syndromes $[22,28]$. The analgesic and anti-inflammatory effects of lavender oil have been demonstrated, and it has been stated that it exerts an opioid-like effect, and its mechanism of action may be related to the activation of ion channels via protein $G$ [22]. It has also been shown that linalool, a monoterpene alcohol found in lavender volatile compounds, activates hypothalamic orexin neurons, which play an important role in pain physiology, significantly increasing the pain threshold and reducing pain behavior [29].

Anxiety is one of the common symptoms of fibromyalgia, and studies have reported that lavender essential oil, especially administered by inhalation, is effective in reducing anxiety levels and can be used in the treatment of chronic anxiety as complementary therapy. It is considered that aromatherapy shows its therapeutic effect both psychologically and through the physiological effects of the volatile compounds it contains, and it is particularly effective in the limbic system. It has also been stated that lavender oil can increase the effect of gamma-aminobutyric acid in the amygdala, with an effect similar to benzodiazepines $[15,20,27]$. In addition, a clinical study investigating the effects of lavender aromatherapy on sleep disorders reported that lavender had a lasting effect on sleep quality and a rebalancing effect on the sleep cycle [21].

In the current study, the SF-36 Scale was preferred because its subscales include components such as general body pain, anxiety, and sleep disturbance. We did not find any clinical study in the literature that used SF-36 to assess these complaints after lavender oil aromatherapy in patients 
TABLE 3: Average values of the SF-36 subscale scores previously reported for the Turkish society [30].

\begin{tabular}{lcc}
\hline Subscales & Female & Male \\
\hline General health & $69.1 \pm 16.9$ & $73.6 \pm 14.9$ \\
Physical functioning & $80.6 \pm 21.7$ & $89.8 \pm 19.3$ \\
Physical role & $82.9 \pm 28.6$ & $89.8 \pm 19.3$ \\
Mental role & $89.0 \pm 22.5$ & $92.8 \pm 15.1$ \\
Social functioning & $90.1 \pm 12.9$ & $91.7 \pm 12.8$ \\
Body pain & $81.0 \pm 20.2$ & $85.1 \pm 16.4$ \\
Mental health & $70.1 \pm 11.4$ & $71.0 \pm 10.6$ \\
Vitality & $63.4 \pm 13.7$ & $65.7 \pm 11.9$ \\
\hline
\end{tabular}

with fibromyalgia, and therefore, our study can be considered a pioneer in this and similar therapies.

When the subscales of SF-36 were evaluated, it was observed that the scores of the general health subscale after aromatherapy did not statistically significantly differ compared to the baseline, and they were also below the average scores (female-male) of the Turkish population identified by Demiral and colleagues as shown in Table 3 [30]. Although there was a statistically significant improvement in the scores of physical functioning, physical role, mental role, social functioning, and body pain subscales, even the postaromatherapy scores in these subscales were below the average scores of the Turkish population for both genders. When the scores of the mental health and vitality subscales were evaluated, there was a statistically significant increase in these scores, and they were similar to the average scores of the Turkish population. It is considered that this improvement may be related to the analgesic, anti-inflammatory, sleepregulating, and anxiety-reducing effects of linalool and linalyl acetate, which are the major volatiles contained in lavender oil. We found no statistically significant increase in the general health subscale scores between the two evaluation times, which can be attributed to the limited number of patients enrolled in the study and the duration of treatment being too short to make a general health assessment.

\section{Conclusions}

After lavender oil aromatherapy, there was a statistically significant increase in the perception of well-being in all subscales, except for the general health subscale, and this increase in the scoring of the mental health and vitality subscales was similar to the average values of the healthy Turkish population. In line with these data, it can be stated that lavender oil aromatherapy can improve the health-related quality of life of patients with fibromyalgia. However, further comprehensive studies are needed to confirm our findings.

\section{Data Availability}

The data used to support the findings of this study are available from the corresponding author upon request.

\section{Conflicts of Interest}

The authors declare that they have no conflicts of interest regarding the publication of this paper.

\section{Acknowledgments}

The authors thank phytotherapist Dr. Sedat Yıldız for his assistance and support. The research and publication of this article were funded by the authors.

\section{References}

[1] D. J. Clauw, "Fibromyalgia," The Journal of the American Medical Association, vol. 311, no. 15, pp. 1547-1555, 2014.

[2] F. Heidari, M. Afshari, and M. Moosazadeh, "Prevalence of fibromyalgia in general population and patients, a systematic review and meta-analysis," Rheumatology International, vol. 37, no. 9, pp. 1527-1539, 2017.

[3] E. Bellato, E. Marini, F Castoldi et al., "Fibromyalgia syndrome: etiology, pathogenesis, diagnosis, and treatment," Pain Research and Treatment, vol. 2012, Article ID 426130, 17 pages, 2012.

[4] A. M. Castro Sánchez, H. García López, M. Fernández Sánchez et al., "Improvement in clinical outcomes after dry needling versus myofascial release on pain pressure thresholds, quality of life, fatigue, pain intensity, quality of sleep, anxiety, and depression in patients with fibromyalgia syndrome," Disability and Rehabilitation, vol. 41, no. 19, pp. 2235-2246, 2019.

[5] "The world health organization quality of life assessment (WHOQOL): position paper from the world health organization," Social Science \& Medicine, vol. 41, no. 10, pp. 1403-1409, 1995.

[6] U. N. Ijoma, N. N. Unaogu, T. I Onyeka et al., "Health-related quality of life in people with chronic diseases managed in a low-resource setting - a study from South East Nigeria," Nigerian Journal of Clinical Practice, vol. 22, no. 9, pp. 1180-1188, 2019.

[7] M. Offenbächer, S. Sauer, N. Kohls, M. Waltz, and P. Schoeps, "Quality of life in patients with fibromyalgia: validation and psychometric properties of the German ruality of life scale (QOLS-G)," Rheumatology International, vol. 32, no. 10, pp. 3243-3252, 2012.

[8] K. Meyer, A. K. Wahl, I. T. Bjørk, T. Wisløff, A. Hartmann, and M. H. Andersen, "Long-term, self-reported health outcomes in kidney donors," BMC Nephrology, vol. 17, no. 1, p. 8, 2016.

[9] H. Koçyigit, S. Gülseren, A. Erol, N. Hizli, and A. Memis, "The reliability and validity of the Turkish version of quality of life questionnaire of the European foundation for osteoporosis (QUALEFFO)," Clinical Rheumatology, vol. 22, no. 1, pp. 18-23, 2003.

[10] I. Bashan, M. Bakman, Y. Uysal, and E. Mert, "Regulation of Dietary Habits: the effect of losing weight on quality of life," Pakistan Journal of Medical Sciences, vol. 34, no. 5, pp. 1253-1256, 2018.

[11] S. Chinn, W. Caldwell, and K. Gritsenko, "Fibromyalgia pathogenesis and treatment options update," Current Pain and Headache Reports, vol. 20, no. 4, p. 25, 2016.

[12] D. J. David and D. Gourion, “Antidépresseurs et tolérance: déterminants et prise en charge des principaux effets indésirables," L'Encéphale, vol. 42, no. 6, pp. 553-561, 2016.

[13] M. M. Aman, R. Jason Yong, A. D. Kaye, and R. D. Urman, "Evidence-based non-pharmacological therapies for fibromyalgia," Current Pain and Headache Reports, vol. 22, no. 5, p. 33, 2018.

[14] A. Nasiri and M. A. Mahmodi, "Aromatherapy massage with lavender essential oil and the prevention of disability in ADL 
in patients with osteoarthritis of the knee: a randomized controlled clinical trial," Complementary Therapies in Clinical Practice, vol. 30, pp. 116-121, 2018.

[15] D. Donelli, M. Antonelli, C. Bellinazzi, G. F. Gensini, and F. Firenzuoli, "Effects of lavender on anxiety: a systematic review and meta-analysis," Phytomedicine, vol. 65, Article ID 153099, 2019.

[16] National Library of Medicine, Drugs and Lactation Database (LactMed), National Library of Medicine (US), Bethesda, MD, USA, 2006.

[17] F. Wolfe, "New American college of rheumatology criteria for fibromyalgia: a twenty-year journey," Arthritis Care \& Research, vol. 62 , no. 5, pp. 583-584, 2010.

[18] A. de Groot and E. Schmidt, "Essential oils, part V: peppermint oil, lavender oil, and lemongrass oil," Dermatitis, vol. 27 , no. 6, pp. 325-332, 2016.

[19] A. B. Mohabbat, S. Mahapatra, S. M. Jenkins, B. A. Bauer, A. Vincent, and D. L. Wahner-Roedler, "Use of complementary and integrative therapies by fibromyalgia patients: a 14-year follow-up study," Mayo Clinic Proceedings: Innovations, Quality \& Outcomes, vol. 3, no. 4, pp. 418-428, 2019.

[20] B. J. Malcolm and K. Tallian, "Essential oil of lavender in anxiety disorders: ready for prime time?," Mental Health Clinician, vol. 7, no. 4, pp. 147-155, 2017.

[21] A. S. Lillehei, L. L. Halcón, K. Savik, and R. Reis, "Effect of inhaled lavender and sleep hygiene on self-reported sleep issues: a randomized controlled trial," The Journal of Alternative and Complementary Medicine, vol. 21, no. 7, pp. $430-438,2015$.

[22] G. L. D. Silva, C. Luft, A. Lunardelli et al., "Antioxidant, analgesic and anti-inflammatory effects of lavender essential oil," Anais da Academia Brasileira de Ciências, vol. 87, no. 2, pp. 1397-1408, 2015 Aug.

[23] A. Salamati, S. Mashouf, F. Sahbaei, and F. Mojab, "Effects of inhalation of lavender essential oil on open-heart surgery pain," Iranian Journal of Pharmaceutical Research: IJPR, vol. 13, no. 4, pp. 1257-1261, 2014 Fall.

[24] J. T. Kim, M. Wajda, G. Cuff et al., "Evaluation of aromatherapy in treating postoperative pain: pilot study," Pain Practice, vol. 6, no. 4, pp. 273-277, 2006.

[25] J. J. Gedney, T. L. Glover, and R. B. Fillingim, "Sensory and affective pain discrimination after inhalation of essential oils," Psychosomatic Medicine, vol. 66, no. 4, pp. 599-606, 2004.

[26] J. Buckle, "Use of aromatherapy as a complementary treatment for chronic pain," Alternative Therapies in Health and Medicine, vol. 5, no. 5, pp. 42-51, 1999.

[27] H. Cavanagh and J. M. Wilkinson, "Biological activities of lavender essential oil," Phytotherapy Research, vol. 16, no. 4, pp. 301-308, 2002.

[28] H. U. Zeilhofer, "Prostanoids in nociception and pain," Biochemical Pharmacology, vol. 73, no. 2, pp. 165-174, 2007.

[29] S. Tashiro, R. Yamaguchi, S Ishikawa et al., "Odour-induced analgesia mediated by hypothalamic orexin neurons in mice," Science Report, vol. 6, p. 37129, 2016.

[30] Y. Demiral, G. Ergor, B. Unal et al., "Normative data and discriminative properties of short form 36 (SF-36) in Turkish urban population," BMC Public Health, vol. 6, no. 1, p. 247, 2006. 